\title{
Post Endoscopic Retrograde Cholangiopancrea-Togram (ERCP) Perforation Managed Successfully Conservatively: A Case Report
}

Abdul Malek Bin Mohamad, Azrin Waheedy Ahmad, Junaini Kasian

Department of Surgery, International Islamic University of Malaysia Medical Centre, Pahang, Malaysia

Introduction: We aim to report an uncommon case of post ERCP perforation that effectively managed conservatively in non-hepatobiliary surgery centre. Case report: A 46-year-old man diagnosed to have obstructive jaundice secondary to distal common bile duct (CBD) stone. He underwent ERCP at a private centre, sphincterotomy was performed, but, the operator had failed to insert the stent and complicated with post ERCP perforation evidenced by contrast extravasation beyond $1 / 3^{\text {rd }}$ of the CBD and referred to our centre. Patient was subjected for re ERCP. There were difficulties in cannulating the CBD and stent was inserted. Cholangiogram revealed contrast leak around the pancreatic duct and bifurcation of hepatic duct. There was no evidence of CBD stone. Computed Tomography (CT) of the abdomen revealed extensive subcutaneous emphysema on the right side of the abdomen to right inguinal region, extensive retroperitoneal free air and pneumoperitoneum, but there were no free fluid or contrast extravasation. The patient subjected for non-operative management (NOM) for the complication and kept fasting with total parenteral nutrition and intravenous antibiotic. He recovered well with the opted management. Patient was programmed with Gastrograffin study after 10 days that showed no evidence of contrast leak to suggest free bowel injury. He was allowed orally after that and was discharged well after 15 days with stent in situ. During follow up, he was well, and the stent removed after 3 months. Patient planned for laparoscopic cholecystectomy and on table cholangiogram. Conclusion: Post ERCP perforation is uncommon but lethal. Early recognition of the complication is crucial hence appropriate management can be arranged to avoid death. To date, surgery is not the only choice available to manage this complication. 